\title{
Artificial Intelligence in the public sector: A study of challenges and opportunities for Nor- wegian municipalities
}

\author{
Patrick Mikalef ${ }^{1,2}$ Siw Olsen Fjørtoft ${ }^{1}$ and Hans Yngvar Torvatn ${ }^{1}$ \\ ${ }^{1}$ SINTEF Digital, S. P. Andersens veg 5 7031, Trondheim, Norway \\ ${ }^{2}$ Norwegian University of Science and Technology, Sem Saelands Vei 7-9 7491, Trondheim, \\ Norway \\ \{patrick.mikalef, siw.fjortoftesintef.no, \\ hans.torvatn\} esintef.no
}

\begin{abstract}
The value of Artificial Intelligence (AI) in augmenting or even replacing human decision-making in the organizational context is gaining momentum in the last few years. A growing number of organizations are now experimenting with different approaches to support and shape their operations. Nevertheless, there has been a disproportionate amount of attention on the potential and value that AI can deliver to private companies, with very limited empirical attention focusing on the private sector. The purpose of this research is to examine the current state of AI use in municipalities in Norway, what future aspirations are, as well as identify the challenges that exist in realizing them. To investigate these issues, we build on a survey study with respondents holding IT management positions in Norwegian municipalities. The results pinpoint to specific areas of AI applications that public bodies intend to invest in, as well as the most important challenges they face in making this transition
\end{abstract}

Keywords: Artificial Intelligence, Business Value, Public Sector, Adoption, Empirical

\section{Introduction}

Artificial Intelligence (AI) can be defined as a set of technologies that simulate human cognitive processes, including reasoning, learning, and self-correction. Recent years have seen an increased interest in the potential uses of AI in private and public organizations [1]. The prevailing argument in applying AI technologies in such organizational settings is that it can enhance, and in some cases even replace, human decision-making and action [2]. This ability provides many opportunities to utilize human resources to more meaningful and less repetitive tasks, while at the same time improving efficiency, reducing errors, and slicing costs [3]. In fact, there have been several publications from academic and popular press to date regarding the potential of AI, which much discussions regarding how it can revolutionize the way organizations do business and interact 
with their customers [4]. Despite much promise however, and a strong wave of enthusiasm regarding the potential of $\mathrm{AI}$, there is still very limited understanding regarding the status of AI adoption, the expectations of organizations, as well as the challenges they face when adopting and deploying such solutions [5]. This issue is particularly apparent in the public sector, where the competitive pressure that typically describes private companies is absent, and deployments are usually less swift [6].

The opportunities of applying AI technologies in the public domain have been documented extensively in several early studies [7]. These range from using chatbots to interact with citizens about procedures and other types of queries [8], to deploying sophisticated methods to identify fraud detection [9], and using autonomous vehicles in traditionally human-executed tasks [10]. Such uses of AI demonstrate that the public sector can benefit in many ways by such technologies when they are applied to key activities that are within the realm of their responsibilities. Nevertheless, despite much promise and a strong emphasis on AI applications from national and European bodies [11], we still have very limited understanding about what the status is in public bodies regarding adoption levels, what IT managers see as the most difficult hurdles to overcomes in order to make such objectives a reality, as well as what is the anticipated impact that AI will have on key performance indicators of public bodies [12]. Studies have shown that when it comes to technology adoption and challenges faced, public and private organizations have to overcome some common challenges but are also faced with distinct differences [13].

Building on this state of knowledge, and on the great promise that AI can produce for public organizations, this study seeks to explore the current level of adoption of AI in different areas of application pertinent to public organization activities. This research also seeks to highlight what are the key challenges public bodies face when routinizing such technologies, and to understand where public organizations see the greatest potential [14]. Exploring these aspects is crucial in order to direct investments and to deploy such technologies without any major hindrances. To empirically explore the research questions, we focus on Norwegian municipalities, and through a recent nation-wide survey present the outcomes from the answers received from IT managers. We selected municipalities as they typically have a broad range of activities that fall under their jurisdiction. Furthermore, the case of Norway is seen as well-suited as the degree of digitalization of public bodies is one of the highest word-wide, therefore being a good indicator of challenges, that other public bodies may face world-wide. The results provide us with an understanding of which are the priority areas that municipalities see as most important to deploy AI solutions, as well as what are the main constraints the currently face. This provides policy makers and practitioners with a clear view of what measures need to be taken to accelerate AI diffusion in the public sector through focused actions.

The rest of the paper is structured as follows. In section 2 we overview the current state of knowledge regarding AI applications and highlight some key areas that have been documented in literature as being of increased significance for public bodies. We then proceed to discuss the context of Norwegian municipalities and the link to strategic direction set by the local and central government. Section 3 outlines the data collection process, the measures used to capture notions, as well as the demographics of 
respondents. Section 4 presents the findings from this exploratory study, illustrating the current state of adoption based on different functional areas, the planned level of deployments, as well as the most important challenges faced in realizing them. We conclude the paper with a discussion of the theoretical and practical implications of this work as well as some suggestions for future research.

\section{Background}

\subsection{Business value of Artificial Intelligence}

Following the emergence of big data analytics and the rapid adoption of Internet-ofThings (IoT), Artificial Intelligence applications have seen a renewed interest building on the massive amounts of data collected and stored. This data has enabled practitioners to realize such AI applications, and grounded on advanced techniques such as deep learning and reinforcement learning. Unlike the previous decades where such massive data was located in the hands of research institutes, nowadays an increasing number of public and private organizations are in place to collect, store and analyze such data as a result of falling storage prices and increased processing capacity. This has sparked a new wave of enthusiasm in practitioners regarding the potential applications and the business value that AI applications can deliver [15]. Much has been written about the increased speed and accuracy that AI can deliver, and the large workload that can be assigned to such technologies. Recent studies emphasize that the role of AI in the contemporary economy is not to completely replace human labor, but rather to take over repetitive and manual tasks. Only by doing so will organizations be able to harness the skills in which humans excel in such as creative thinking and problem solving. Furthermore, other recent articles see AI and human cooperating harmoniously in a synergy called augmented intelligence. According to this view, AI applications can help enhance human tasks, automating many activities and serving as decision-making aids. Through this way organizations will be able to harness the complementary strengths of humans and machines.

Despite these claims, research on the business potential of AI is still at a very early stage $[5,16]$. This is especially evident in relation to the public sector, which typically lags in technological adoption in comparison the private sector. To date, most academic literature has focused on potential applications of $\mathrm{AI}$ in the realm of public administration. For instance, a prominent example is that of chatbots for interactions with citizens, whereby human capacity to solve queries is automated. In another study, the use of AI for autonomous vehicles is examined, in which the task of internal resource management and delivery is assigned to unmanned vehicles or robots. Anecdotal claims suggest that such investments can reduce time needed to supple units with necessary resources, reduce human errors, and slice down costs. This application of AI has significant value in public institutions such as hospitals and medical centers, where consumables and critical equipment can be delivered on demand and without the need of human resources to be committed for such tasks. Extending on this, there has been a large discussion about the vast opportunities that open up with utilizing personnel in more 
meaningful and important tasks. Another important area where AI has been suggested to produce value for public administration is to automatize financial processes and detect fraud. Advanced techniques of vision computing are able to scan thousands of documents in a matter of seconds, register details of expenses and other financial information, and detect anomalies or potential fraud. In this way, AI not only contributes to increased speed in handling such financial information but is also less prone to human error in detecting errors or cases of potential fraud. While the academic and practicebased literature stresses the value of AI in tasks of public administration, to date we have little empirical evidence about if public bodies are actually deploying such solutions, and if so where. Furthermore, there is very little research regarding the plans of public organizations and which areas they see as the most likely to strengthen by means of AI investments. In the following sub-section, we discuss about the context of Norwegian municipalities and the strategic and operational decisions that guide their actions.

\subsection{The context of Norwegian municipalities and digitalization}

The municipality is the lowest administrative and electoral level in Norway. Currently, there are 422 municipalities and size vary from a couple of hundred inhabitants to over 650,000 in Oslo. In terms of area, you have the same variation, the smallest municipality is $6 \mathrm{~km} 2$ and the largest is $9700 \mathrm{~km} 2$. Regardless of population or area, the municipalities must provide an equal service to the population. The municipalities have a diverse responsibility, ranging from basic welfare benefits such as schooling, kindergarten, social and medical assistance, child welfare, nursing home; to local roads, water, refuse collection, fire and rescue services; furthermore, the maintenance of churches and cemeteries, and cultural services such as operation of the cinema. Norway is focusing on good digital infrastructure. The Government has set a goal that by the year 2020, 90 per cent of the households in the country will have at least $100 \mathrm{Mbit} / \mathrm{s}$ offer based on commercial development in the market. In the long term, the goal is for all households to have offers for high-speed broadband, also in areas where the population is low and less profitable with development. Expansion of access to fiber is also in full swing, in addition to mobile networks. From autumn 2019, the 5G technology is widely available to residents of Trondheim municipality, and $5 \mathrm{G}$ networks have been prepared in ten major Norwegian cities from 2020.

Internet access and bandwidth is a prerequisite for citizens and businesses to use public digital services. In addition, signals from sensors, welfare technology, traffic monitoring, and digital exchange of information, such as a «patient ready to leave hospital» message, have become an integral part of municipal service production. In recent years, there has been an increased support for common digitization solutions for Norwegian municipalities. An example is SvarUt, which is a solution that conveys documents between sender and recipient via different channels. The municipalities and county authorities have had access since 2013. By the end of 2018, more than 400 municipalities, but also all county municipalities and 77 government agencies had adopted the solution. In 2018, approximately 7.5 million letters were sent through SvarUt in Norwegian 
municipalities and county municipalities. The potential for the number of shipments through SvarUt within one year is estimated at ten per capita, or approx. 53 million. But this is only one of many solutions to meet demands of more efficient municipal service production.

Three new common digitization solutions were offered to the municipalities in autumn 2018, DigiSos - a solution for being able to apply digitally for financial social assistance, DigiHelse - a solution that enables citizens to see agreements and have dialogue with the home service, and Minside- a service that provides residents access to all matters that they have with the municipality or the county municipality. These examples indicate that Norwegian municipalities to some extent have started a common digitalization to better citizen services. In our study we aim to examine the digital maturity and readiness for next level technologies, such as AI. One final thing that is worth taking note of is the ongoing merging of Norwegian municipalities. The Government has initiated a municipal reform in which 119 municipalities will be 47 new. This means a reduction from 422 to 356 municipalities from January 2020. More inhabitants per municipality and larger geographical distances can increase the demands of good digital services to the inhabitants - A factor that might promote further innovation in municipal service production.

\section{$3 \quad$ Method}

\subsection{Sampling and data collection}

To explore the questions raised in this study, we built on a survey-based research method aimed at key respondents within Norwegian municipalities. Key respondents included heads of the IT division, IT managers, and senior data scientists. The rationale of selecting respondents in these positions was that they are the most knowledgeable about the current status of AI investments in their organizations and are also the best informed about future areas of interest as well as challenged faced during implementations. To identify appropriate respondents, a search was conducted on the webpages of municipalities in Norway. These provide contact details for employees at different roles within the IT department, allowing us to locate those that fit the profile we were looking for better. A list of 83 respondents was compiles including some of the largest municipalities in Norway. Invitations were sent out to respondents via email, with a link to an online questionnaire. The questionnaire was designed in such a way so that it did not require more than 15 minutes to be filled out, and covered several areas relating to AI investments and future plans. After the initial invitation, two reminders were sent out within a two-week interval. The total number of complete responses received from this sample was 46 , with 9 others being incomplete, and thus not retained for further analysis. 


\subsection{Sample demographics}

The final sample differed in several aspects, which provided an interesting dataset for further analysis. As planned, respondents held positions of senior management within the IT department, with the most frequent title being IT manager, digital transformation director, and department leader. With regards to AI adoption, the largest proportion of respondents indicated that their organization has not yet adopted AI, while from the rest, $10.9 \%$ had just started deploying AI within the year and another $19.6 \%$ had been using AI for 1-2 years. These results demonstrate that AI adoption in municipalities of Norway is still very low, and even those that have adopted some form of AI have only done so fairly recently. With regards to size, the sample varied significantly, with the largest proportion being municipalities with 500-1.000 employees, followed by a slightly smaller size-class of 100-500 (23.9\%), while there were also some responses of the large municipalities with over 5.000 employees (13.0\%). Respectively, the IT departments were fairly small, with the largest proportion being between 1-9 people $(65.2 \%)$, followed by IT departments with $10-49$ employees $(26.1 \%)$, and a small number with 50-249 people (8.7\%). These demographics are in alignment with the distribution of Norwegian municipalities, where there are primarily small municipalities and IT departments, and a smaller number of large ones (e.g. Oslo and Trondheim).

Table 1. Sample demographics

\begin{tabular}{lll}
\hline Factors & Sample (N) & Proportion (\%) \\
\hline Years using AI & 31 & $67.4 \%$ \\
We do not use AI yet & 5 & $10.9 \%$ \\
Less than 1 year & 9 & $19.6 \%$ \\
1-2 years & 1 & $2.2 \%$ \\
2-3 years & & \\
& & \\
How many people work in your organization? & 3 & $6.5 \%$ \\
Less than 100 & 11 & $23.9 \%$ \\
100-500 & 14 & $30.4 \%$ \\
500-1.000 & 8 & $17.4 \%$ \\
1.000-2.500 & 4 & $8.7 \%$ \\
2.500-5.000 & 6 & $13.0 \%$ \\
More than 5.000 & & \\
How many people work in the IT department & & \\
1-9 & 30 & $65.2 \%$ \\
10-49 & 12 & $26.1 \%$ \\
50-249 & 4 & $8.7 \%$ \\
More than 250 & 0 & $0.0 \%$ \\
\hline
\end{tabular}




\section{$4 \quad$ Results}

To analyze data, we used descriptive statistics and paired sample t-tests that were run through the software package IBM SPSS. The first part of the analysis involved examining the current state of AI use within municipalities. In order to examine potential areas of application within the context of public administration, we reviewed the relevant literature that discusses the most promising ways in which AI can be leveraged $[17,18]$. The results depicted below illustrate the percentage of municipalities within our sample that have not used AI in the specific areas of application, as well as those that have initiated deployments. From the results depicted in the graph below we can see that the most popular applications of AI for municipalities in Norway include intelligent interaction agents with citizens $(28.9 \%)$, real-time translations for meetings including speech-to-speech and speech-to-text (21.1\%), as well as request processing and applications handling and automatizing data entry with $15.7 \%$ each. These results demonstrate a clear trend in using AI to interact with citizens primarily, and in assisting in tasks where additional aid is required, such as communication. On the other hand, there is a large proportion of municipalities that have still not adopted any form of AI.

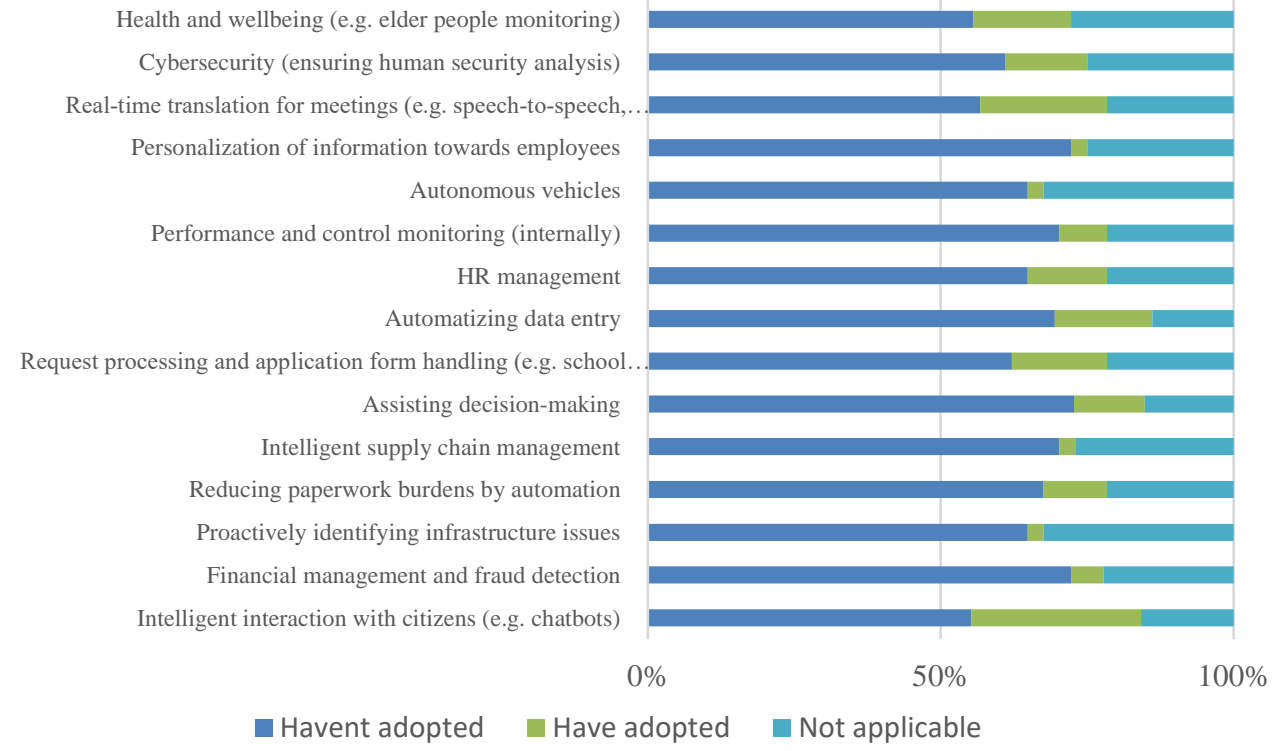

Fig. 1. Current levels of AI adoption in Norwegian municipalities by area of use

Extending on these findings, the next series of questions asked respondents to report the degree to which they plan to use AI in the future in the abovementioned areas. The 
reason for asking responses to evaluate future plans is to try to comprehend the types of investments they would be required to make and to propose policies and roadmaps to achieve desired objectives. Furthermore, future investment plans also are a good indication about where other public bodies or municipalities in other countries plan to increase their investments. To examine this, we asked respondents to whom these areas were applicable to assess the level to which they are planning to invest in AI technology to support them. Responses were marked on a 7-point Likert scale, with 1 denoting a very low intention to adopt, while 7 indicates a very high intention to implement AI for the particular task. From the results it is evident that some areas of AI use are of increased interest for municipalities. First and foremost, the use of intelligence interaction with citizens in the form of chatbots is regarded by respondents as a top priority to invest in for the near future. It is a frequent phenomenon that employees are overloaded with such requests and queries that can disrupt work and slow down other critical tasks. Since a lot of information on municipality websites requires effort to find, the use of chatbots to locate and provide this information in an easy way can free up the need for human resources in this task while at the same time provide increase citizen satisfaction with services. Similarly, request processing and application form handling was the area with the second highest interest for future adoption, with health and wellbeing services ranking as third. These results provide a clear view of where municipalities see the most value from $\mathrm{AI}$ in relation to the tasks that is within their realm of responsibilities. It also serves as a good roadmap to direct policy and funding to support such actions.

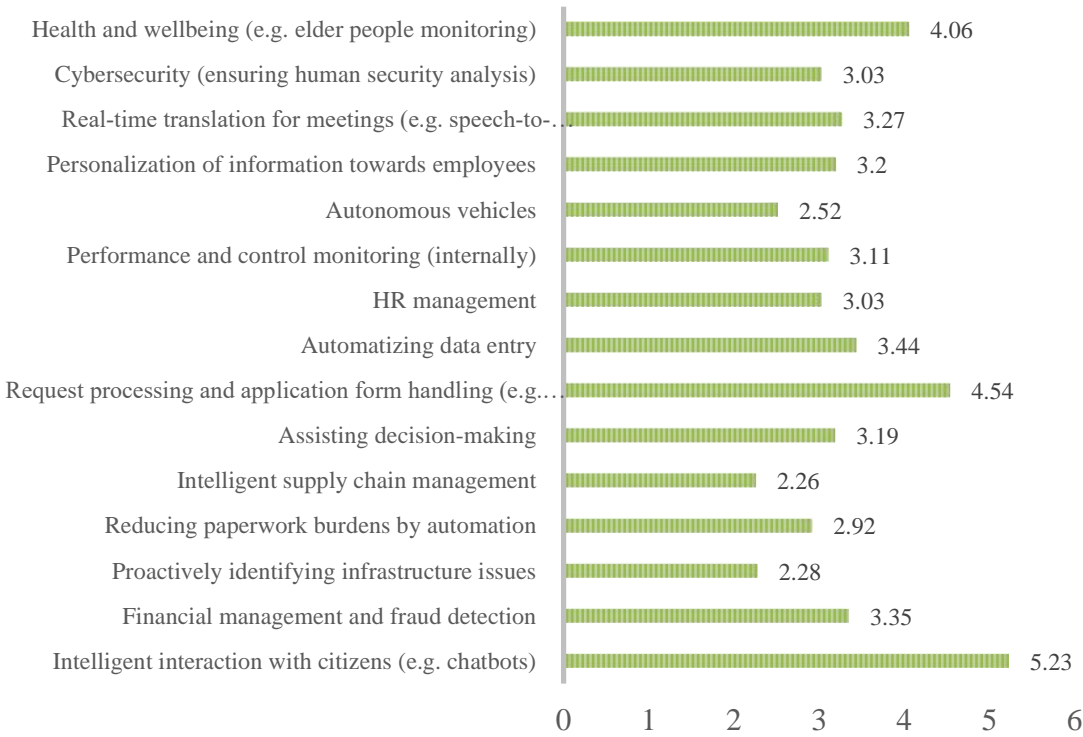

Fig. 2. Intention to adopt AI in Norwegian municipalities by area of use 
The final part of our examination looked at the challenge's municipalities face when they adopt AI, or barriers that do not allow them to implement such solutions. In the categories presented below in Fig. 3, we asked respondents to evaluate on a 7-point Likert scale the extent to which each of the categories mentioned bellow was a significant barrier to adopt or implement AI technologies in their everyday operations. The scale ranged from 1, denoting that the specific factor was not an important inhibitor of adoption, to 7 which meant that it was an important hindering factor for implementing AI technologies. The results highlight that the challenges that are faced from municipalities range from both technical to organizational. The most important hindering factor is the inability to integrate systems and data, as well as to ensure that quality data are utilized to train AI. Almost as equally important are organizational factors including the lack of expertise with the necessary know-how, the limited financial resources, as well as organizational inertia. These outcomes demonstrate that if Norwegian municipalities aim to adopt AI, they need both structural reforms as well as financial capital to pursue these directions. In fact, respondents noted that the perception that $\mathrm{AI}$ is not necessary for public administration is not an issue for non-adoption, indicating that low levels of maturity to date are mostly a result of organizational or technical hindrances.

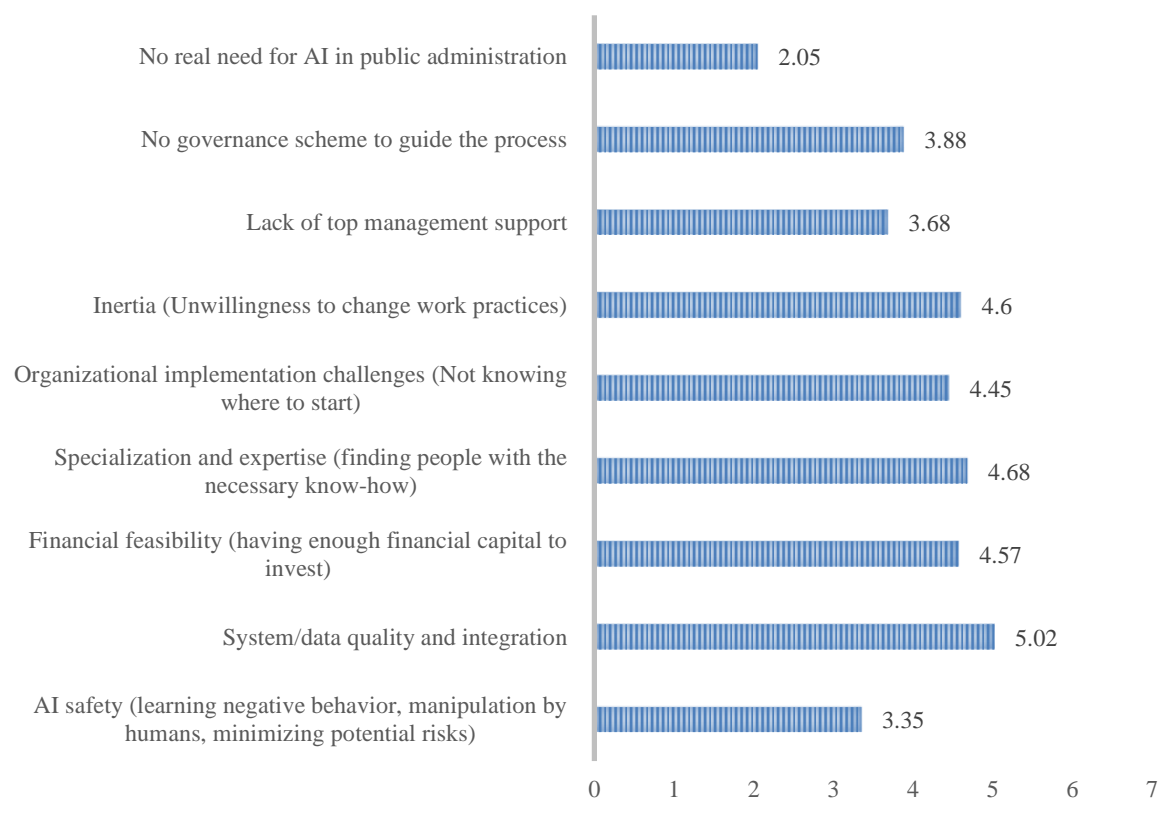

Fig. 3. Challenges in implementing and adopting AI technologies in Norwegian municipalities

\section{Discussion}

Artificial Intelligence has gained eminence over the past few years as a set of technological innovations that can potentially disrupt private and public organizations. There 
is a growing number of research and popular press articles claiming that AI will radically change the way we do business. Despite such claims about the potential of AI there is still very little empirical research concerning the level of use within organizations, and particularly for public bodies. The purpose of this study was to shed some light about the use and intended use of AI within public administration and particularly in Norwegian municipalities. Since the scope of activities of municipalities is to a large extent similar world-wide, areas of using AI, as well as challenges faced during adoption, or even barriers to adoption are likely to be the same also in other countries. To examine these issues, we conducted a survey study with a sample of municipalities in Norway, using senior IT managers as key respondents.

From a theoretical point of view our study contributes to literature by demonstrating the areas which public administration organizations such as municipalities are currently investing in or are planning to do so in the near future. It is important to understand in which areas AI will be central in in order to propose optimal methods for deploying such solutions and streaming adoption processes. As with any technological adoption, inertial forces and challenges are likely to delay implementation or reduce potential business value if appropriate measures are not taken at early stages of projects [19]. Therefore, such cases may serve as lessons learnt for future ventures where organizational know-how can be transferred and best-practices adopted. Furthermore, from a citizen's perspective, knowing what services would be best automated through AI can help create interfaces and AI-supported services that best fulfil citizen needs. It is critical to get feedback from users of these services in order not only to train AI applications but to design the provided services in a way that optimize satisfaction and easeof-use. Furthermore, by knowing what the main challenges are it is possible to develop governance plans and strategies to facilitate adoption, use, and routinization of AI within the public administration sphere. While such governance practices are quite common for other types of information systems, in AI they are still non-existent.

From a practice-based perspective the outcomes of this study can help practitioners identify what the common challenges that they face are and form a knowledge exchange group [20]. Transferring such knowledge to other municipalities, and particularly those that are of a smaller size and with less resources can be particularly beneficial if they want to keep up with new emerging technologies. Furthermore, the outcomes can be used by policy makers in order to direct funding and assist municipalities with their deployments. With AI becoming an increasingly integral part of public administration, it is important that financial resources and policies are put into the right directions. Using such outcomes can help policy makers devise future frameworks and develop assistance plans for public bodies.

While this study used a small sample of Norwegian municipalities it does provide some insight of trends. We intend to expand the sample of municipalities and also survey other public bodies to examine the level of adoption and challenged faced. Furthermore, in extended reach the plan is to examine the readiness level of Norwegian municipalities in greater detail so that more concrete action plans can be formulated, and specific recommendations can be provided. We also encourage future researchers to examine how AI can be used to transform operations of public administration, and how deployments can be optimized in order to maximize derived value. It is important to 
understand how such novel technologies can shape and complement existing strategies [21].

\section{References}

1. Ransbotham, S., Gerbert, P., Reeves, M., Kiron, D., Spira, M.: Artificial Intelligence in Business Gets Real. MIT sloan management review (2018)

2. Davenport, T.H., Ronanki, R.: Artificial intelligence for the real world. Harvard business review 96, 108-116 (2018)

3. Li, B.-h., Hou, B.-c., Yu, W.-t., Lu, X.-b., Yang, C.-w.: Applications of artificial intelligence in intelligent manufacturing: a review. Frontiers of Information Technology \& Electronic Engineering 18, 86-96 (2017)

4. Huang, M.-H., Rust, R.T.: Artificial intelligence in service. Journal of Service Research 21, 155-172 (2018)

5. Mikalef, P., Boura, M., Lekakos, G., Krogstie, J.: Big data analytics and firm performance: Findings from a mixed-method approach. Journal of Business Research 98, 261-276 (2019)

6. Sun, T.Q., Medaglia, R.: Mapping the challenges of Artificial Intelligence in the public sector: Evidence from public healthcare. Government Information Quarterly 36, 368-383 (2019)

7. Wirtz, B.W., Müller, W.M.: An integrated artificial intelligence framework for public management. Public Management Review 1-25 (2018)

8. Park, D.-a.: A Study on Conversational Public Administration Service of the Chatbot Based on Artificial Intelligence. Journal of Korea Multimedia Society 20, 1347-1356 (2017)

9. Herrera, J.L.L., Figueroa, H.V.R., Ramírez, E.J.R.: Deep fraud. A fraud intention recognition framework in public transport context using a deep-learning approach. In: 2018 International Conference on Electronics, Communications and Computers (CONIELECOMP), pp. 118-125. IEEE, (Year)

10.Hengstler, M., Enkel, E., Duelli, S.: Applied artificial intelligence and trust-The case of autonomous vehicles and medical assistance devices. Technological Forecasting and Social Change 105, 105-120 (2016)

11.Cath, C., Wachter, S., Mittelstadt, B., Taddeo, M., Floridi, L.: Artificial intelligence and the 'good society': the US, EU, and UK approach. Science and engineering ethics 24, 505-528 (2018) 12.Goolsbee, A.: Public policy in an AI economy. National Bureau of Economic Research (2018) 13.Liu, S.M., Yuan, Q.: The evolution of information and communication technology in public administration. Public Administration and Development 35, 140-151 (2015)

14.Mikalef, P., Van de Wetering, R., Krogstie, J.: Big Data enabled organizational transformation: The effect of inertia in adoption and diffusion. In: Business Information Systems (BIS). (Year)

15.McAfee, A., Brynjolfsson, E.: Machine, platform, crowd: Harnessing our digital future. WW Norton \& Company (2017)

16.Mikalef, P., Boura, M., Lekakos, G., Krogstie, J.: Big data analytics capabilities and innovation: the mediating role of dynamic capabilities and moderating effect of the environment. British Journal of Management 30, 272-298 (2019) 
17.Duan, Y., Edwards, J.S., Dwivedi, Y.K.: Artificial intelligence for decision making in the era of Big Data-evolution, challenges and research agenda. International Journal of Information Management 48, 63-71 (2019)

18.Mikhaylov, S.J., Esteve, M., Campion, A.: Artificial intelligence for the public sector: opportunities and challenges of cross-sector collaboration. Philosophical Transactions of the Royal Society A: Mathematical, Physical and Engineering Sciences 376, 20170357 (2018)

19.Mikalef, P., Krogstie, J.: Big Data Governance and Dynamic Capabilities: The Moderating effect of Environmental Uncertainty. Pacific Asia Conference on Information Systems (PACIS). AIS, Yokohama, Japan (2018)

20.Mikalef, P., Framnes, V.A., Danielsen, F., Krogstie, J., Olsen, D.H.: Big Data Analytics Capability: Antecedents and Business Value. In: Pacific Asia Conference on Information Systems. (Year)

21.Mikalef, P., Pateli, A.: Information technology-enabled dynamic capabilities and their indirect effect on competitive performance: Findings from PLS-SEM and fsQCA. Journal of Business Research 70, 1-16 (2017) 